\title{
Pituitary adenoma or pituitary neuroendocrine tumor: a narrative review of controversy and perspective
}

\author{
Xiaohai Liu ${ }^{1,2}$, Renzhi Wang ${ }^{2,3}$, Mingchu Li ${ }^{1,2}$, Ge Chen ${ }^{1,2}$ \\ ${ }^{1}$ Department of Neurosurgery, Xuanwu Hospital Capital Medical University, Beijing, China; ${ }^{2}$ Chinese Pituitary Specialists Congress, Beijing, China; \\ ${ }^{3}$ Department of Neurosurgery, Peking Union Medical College Hospital, Chinese Academy of Medical Sciences and Peking Union Medical College, \\ Beijing, China \\ Contributions: (I) Conception and design: X Liu, G Chen; (II) Administrative support: G Chen, R Wang; (III) Provision of study materials or patients: \\ X Liu, R Wang, M Li, G Chen; (IV) Collection and assembly of data: X Liu, R Wang, M Li, G Chen; (V) Data analysis and interpretation: X Liu, R \\ Wang, M Li, G Chen; (VI) Manuscript writing: All authors; (VII) Final approval of manuscript: All authors. \\ Correspondence to: Ge Chen. Department of Neurosurgery, Xuanwu Hospital Capital Medical University, Beijing100053, China. \\ Email: chengecn@139.com.
}

\begin{abstract}
In 1932, Harvey Cushing first proposed pituitary adenoma (PA) as the term for the cause of acromegaly. After nearly 90 years of research, PA is recognized as the second most common intracranial neoplasm. Most PAs can be easily cured or controlled by conventional therapies, including surgery, medical treatment or radiotherapy. However, nearly $40 \%$ of PAs are invasive, and some adenomas show aggressive behavior, with rapid growth patterns and resistance to conventional treatments, which leads to a poor prognosis; this type of adenoma is called aggressive or refractory PA. For a very small proportion of PAs (approximately $0.2 \%$ ), subarachnoid or systemic metastasis may develop, at which point it is considered malignant and called pituitary carcinoma. Based on distinctions regarding the biological behaviors of adenoma, aggressive adenoma and pituitary carcinoma, the International Pituitary Pathology Club in 2016 formally proposed new terminology for PA: pituitary neuroendocrine tumor (PitNET). Over the last three years, an increasing number of relevant articles have used the term PitNET in place of PA. Interestingly, the fourth edition of the WHO classification of PA released in 2017 did not adopt this nomenclature, and the Pituitary Society published a paper in 2019 suggesting that the new nomenclature does not improve clinical diagnosis or treatment and confuses patients more than the PA terminology. The Pituitary Society insists on using the term PA, as PAs with aggressive behavior only account for a very small proportion of all PAs. Overall, the new terminology may cause significant and unnecessary patient anxiety and confusion. Here, we describe the current perspectives and bases of the two naming methods and offer our opinions on maintaining the original naming system for PA. Nevertheless, early diagnosis and intensive treatment of aggressive PAs is extremely important for improving patient prognosis, regardless of the nomenclature.
\end{abstract}

Keywords: Refractory pituitary adenoma (refractory PA); aggressive pituitary adenoma; pituitary carcinoma; pituitary neuroendocrine tumor (PitNET)

Submitted Dec 16, 2020. Accepted for publication Feb 26, 2021.

doi: $10.21037 /$ tcr-20-3446

View this article at: http://dx.doi.org/10.21037/tcr-20-3446

\section{Introduction}

Pituitary adenoma (PA) is the second most common intracranial tumor, accounting for approximately $15 \%$ of all intracranial tumors, and its incidence rate is increasing yearly (1). In 1932, Harvey Cushing proposed PA as the term to describe the cause of acromegaly (2). After nearly 90 years of research, PAs have been confirmed as monoclonal entities originating from the adenohypophysis (3). Most PAs are benign tumors that can be easily cured or controlled by conventional therapies, including surgery, medical treatment 
or radiotherapy (4). However, a small set of PAs manifesting as a radiologically invasive tumor with an unusually rapid growth rate or clinically relevant growth despite optimized standard therapies (surgery, radiotherapy and conventional medical treatment) should be considered as aggressive PAs, though the prevalence remains unknown (5-7). In general, the invasiveness of pituitary tumors does not always correlate with aggressiveness. Intriguingly, subarachnoid or systemic metastasis develops in a very small number of PAs (approximately $0.2 \%$ ); such a malignancy is called pituitary carcinoma (8). The International Pituitary Pathology Club has formally proposed changing the term PA to pituitary neuroendocrine tumor (PitNET) mainly based on the aggressiveness of some PAs and according to origin of the tumor and the distinctions in biological behavior between adenoma, aggressive adenoma and pituitary carcinoma (9). However, this designation was not adopted in the fourth edition of the WHO classification of PAs published in 2017 (10), and the Pituitary Society published a paper in 2019 suggesting that the new terminology does not improve clinical diagnosis and treatment and confuses patients more than PA terminology (11). Indeed, the Pituitary Society insists on using the term PA as "PAs with aggressive biological behaviors" and "pituitary carcinomas", which account for only a very small proportion of all PAs, suggesting that the new terminology may cause significant and unnecessary patient anxiety and confusion $(11,12)$. Here, we describe the starting point and basis of each naming system. Regardless of the nomenclature, early diagnosis and intensive treatment of aggressive PAs are extremely important for improving patient prognosis. We present the following article in accordance with the Narrative Review reporting checklist (available at http:// dx.doi.org/10.21037/tcr-20-3446).

\section{The proposal of PitNET nomenclature}

As early as 1990, Herman and colleagues confirmed that PAs are monoclonal benign tumors arising from adenohypophyseal cells (13). PAs exhibit a variety of endocrinological clinical manifestations, complex tumorigenesis mechanisms, and obvious intratumor heterogeneity (14). Although most PAs exhibit benign growth patterns and have good prognoses, a small number show aggressive behaviors, with rapid tumor growth patterns and a poor prognosis even with combined therapies, including surgery, routine drug therapy and radiotherapy. For the early diagnosis of such PAs with aggressive behavior and a poor prognosis, the third edition of the WHO classification of PAs released in 2004 defined atypical PA as $\mathrm{PA}$ with a $\mathrm{Ki}-67$ labeling index $>3 \%$, increased mitotic count and p53 loss (15); this type accounts for approximately $2.7-15 \%$ of all PAs (16). However, further studies have found that a diagnosis of atypical PA does not indicate the biological behavior and prognosis of the tumor (17). Therefore, the terminology of atypical PA was abandoned in the fourth edition of the WHO classification of PAs released in 2017, and new nomenclature of "aggressive pituitary adenoma" was proposed; this type of tumor is characterized by rapid growth, radiological invasion, a high Ki-67 proliferation index and refractoriness to conventional therapies, including surgery, traditional drug therapy and radiotherapy (10). According to European Society of Endocrinology Clinical Practice Guidelines for the management of aggressive pituitary tumors and carcinomas, uncontrolled tumor enlargement and/or hormone oversecretion will seriously affect the quality of life or even endanger the life of the patient (5). Temozolomide (TMZ) is currently the only agent that has shown promising results when used as a salvage therapy, though confirmation of this finding in prospective, randomized, controlled trials is lacking (18). Based on definitions in the field of oncology, "tumor" is a general term for benign and malignant tumors, whereas adenoma refers to a benign tumor produced by epithelial cells of a gland. With the shift in terminology to pancreatic NETs, the change from "adenoma" to "tumor" was employed to recognize the lack of predictability and reflect the potential for malignant behavior of pancreatic NETs. Therefore, the International Pituitary Pathology Club in 2016 in France at their 14th meeting formally proposed changing the term from PA to PitNET. PAs display a series of transitions from benign to malignant biological behavior, and these transitions can lead to severe clinical symptoms and a poor prognosis, even if there is no shift to pituitary carcinoma (9). The term tumor emphasizes the unpredictable malignant behavior of some PAs. Moreover, as the adenohypophysis cells secreting pituitary hormones in aggressive PA are neuroendocrine cells, it has been suggested that the term be changed from PA to PitNET. This recommendation has also been recognized by the WHO Consensus Expert Panel in a proposal on the framework for neuroendocrine tumors (19).

\section{The question of the nomenclature of PitNETs}

In 2019, the Pituitary Society published a paper 
Table 1 The differences of the meanings between PAs and PitNETs

\begin{tabular}{lll}
\hline Characteristics & PAs & PitNETs \\
\hline Origin & Adenocyte origin & Specialized cells with traits of both endocrine cells \\
Behavior & Not predictive & Indicating aggressive biological behavior \\
Prognosis & Not predictive & Indicating on poor prognosis \\
Patient & Familiar & Not familiar and may bring anxiety and frustration \\
\hline
\end{tabular}

suggesting that compared with $\mathrm{PA}$ nomenclature, the new nomenclature can neither promote clinical diagnosis and treatment nor reduce confusion in patients (11). Furthermore, the International Pituitary Pathology Club argued that the term PitNET emphasizes the aggressive behavior of some tumors (20). Base on a meta-analysis of radiology and pathology data from autopsy reports, pituitary lesions were incidentally found in $16.7 \%$ of the population (21), and recent cross-sectional, communitybased studies estimated the prevalence of PAs at 78-94 cases per 100,000 population $(22,23)$. In addition, a recent study reported that the prevalence and incidence rates of PAs have increased to approximately 1 in 1,000 with the popularity of magnetic resonance imaging examination (24). Nonetheless, most adenomas are asymptomatic and found incidentally, which are called incidentalomas. These incidentalomas remain asymptomatic during long-term follow-up and do not require treatment. Only a small proportion of PAs are clinically relevant, causing symptoms through oversecretion of hormones or mass effects; these PAs also do need treatment. Most PAs are characterized by benign tumor biological behavior, whereas all NETs are considered malignant tumors (https://www.cancer.net/cance r-types/neuroendocrine-tumors/introduction), as defined by the American Society of Clinical Oncology (12). Overall, the new term PitNET may cause misunderstanding and unnecessary concern in patients; the term emphasizes the aggressiveness of some PAs, and it may not be appropriate, as aggressive tumors are very rare.

Additionally, according to the medical textbook definition, tumor is a nonspecific term that refers to abnormal growth of cells that can either be benign or malignant, and this terminology does not reveal that PA originates from the glandular structures of epithelial tissues of the pituitary gland and is therefore pathologically inappropriate. As defined by the American Society of Clinical Oncology, NETs originate from specialized cells with traits of both hormone-producing endocrine cells and nerve cells. The Mayo Clinic has a similar definition (https://www.mayoclinic.org/diseasesconditions/neuro endocrinetumors/symptomscauses/syc20354132). Both of these definitions suggest that NETs can occur in any part of the body, such as the lungs, gastrointestinal tract, pancreas, and adrenal gland, which are common sites, but without mention of the pituitary gland. Hence, there is no theoretical basis for defining PitNET.

\section{Our perspective}

We are aware that PA is not a simple resectable benign tumor but a complex neuroendocrine disease with a very high incidence. Compared to "large and invasive neoplasms that cause significant morbidity", pituitary incidentalomas not requiring intervention are far more common. Therefore, it is questionable to change the nomenclature of PA that has been used for nearly 90 years to PitNET, which is prone to ambiguity. Our reasons are as follows (Table 1):

(I) The PitNET nomenclature fails to indicate the adenocyte origin and ignores the endocrine background of PAs, which may challenge the classification of tumors of the endocrine system.

(II) The basis for PitNET is the malignant biological behavior and poor prognosis of aggressive PAs. However, the vast majority of PAs are silent and need no clinical intervention. Aggressive PAs with malignant biological behavior are extremely rare. The new term PitNET may bring anxiety and frustration to patients. Both the fourth edition of the WHO PA classification and the Guidelines of the European Endocrine Society for the treatment of aggressive PAs emphasize the malignant biological behavior of a small subset of tumors and suggest early diagnosis and intensive treatment for this subset. Thus, there is no need to develop new nomenclature to emphasize this.

(III) As defined by the American Society of Clinical 
Oncology, NETs are derived from specialized cells with traits of both hormone-producing endocrine cells and nerve cells and occur in common sites such as the lung, gastrointestinal tract, pancreas and adrenal gland, without mention of the pituitary gland. Therefore, the new term PitNET for PA lacks theoretical support.

\section{Conclusions}

As the new term PitNET will create additional ambiguity regarding the origin of PAs and cause anxiety and frustration in patients with typical PAs, we suggest maintaining the term PA and not using PitNET at this time.

\section{Acknowledgments}

Funding: Financial support for this study was provided by the Scientific Research Project of Capital Health Development in 2018 (grant number: 2018-4-4018). The funding institutions played no role in the design of the study, data collection or analysis, decision to publish, or preparation of the manuscript.

\section{Footnote}

Reporting Checklist: The authors have completed the Narrative Review reporting checklist. Available at http:// dx.doi.org/10.21037/tcr-20-3446

Peer Review File: Available at http://dx.doi.org/10.21037/tcr20-3446

Conflicts of Interest: All authors have completed the ICMJE uniform disclosure form (available at http://dx.doi. org/10.21037/tcr-20-3446). The authors have no conflicts of interest to declare.

Ethical Statement: The authors are accountable for all aspects of the work in ensuring that questions related to the accuracy or integrity of any part of the work are appropriately investigated and resolved.

Open Access Statement: This is an Open Access article distributed in accordance with the Creative Commons Attribution-NonCommercial-NoDerivs 4.0 International License (CC BY-NC-ND 4.0), which permits the noncommercial replication and distribution of the article with the strict proviso that no changes or edits are made and the original work is properly cited (including links to both the formal publication through the relevant DOI and the license). See: https://creativecommons.org/licenses/by-nc-nd/4.0/.

\section{References}

1. Melmed S. Pituitary-Tumor Endocrinopathies. N Engl J Med 2020;382:937-50.

2. Cushing $\mathrm{H}$. The basophil adenomas of the pituitary body and their clinical manifestations (pituitary basophilism). 1932. Obes Res 1994;2:486-508.

3. DeLellis RA, Lloyd RV, Heitz PU, et al. Pathology and Genetics of Tumours of Endocrine Organs. Lyons, France: IARC Press, 2004.

4. Molitch ME. Diagnosis and treatment of pituitary adenomas: a review. JAMA 2017;317:516-24.

5. Raverot G, Burman P, McCormack A, et al. European Society of Endocrinology Clinical Practice Guidelines for the management of aggressivepituitary tumours and carcinomas. Eur J Endocrinol 2018;178:G1-G24.

6. Dai C, Feng M, Liu X, et al. Refractory pituitary adenoma: a novel classification for pituitary tumors. Oncotarget 2016;7:83657-68.

7. Dai C, Liu X, Ma W, et al. The Treatment of Refractory Pituitary Adenomas. Front Endocrinol (Lausanne) 2019;10:334.

8. Scheithauer BW, Gaffey TA, Lloyd RV, et al. Pathobiology of pituitary adenomas and carcinomas. Neurosurgery 2006;59:341-53; discussion 341-53.

9. Asa SL, Casar-Borota O, Chanson P, et al. From pituitary adenoma to pituitary neuroendocrine tumor (PitNET): an Inter- national Pituitary Pathology Club proposal. Endocr Relat Cancer 2017;24:C5-C8.

10. WHO (2017). WHO classification of tumours of endocrine organs. WHO classification of tumours.

11. Ho KKY, Fleseriu M, Wass J, et al. A tale of pituitary adenomas: to NET or not to NET: Pituitary Society position statement. Pituitary 2019;22:569-73.

12. Ho KKY, Fleseriu M, Wass J, et al. The tale in evolution: clarity, consistency and consultation, not contradiction and confusion. Pituitary 2020;23:476-7.

13. Herman V, Fagin J, Gonsky R, et al. Clonal origin of pituitary adenomas. J Clin Endocrinol Metab 1990;71:1427-33.

14. Melmed S. Pathogenesis of pituitary tumors. Nat Rev Endocrinol 2011;7:257-66.

15. WHO (2004). Pathology and genetics of tumours of 
endocrine organs. WHO classification of tumours.

16. Zada G, Woodmansee WW, Ramkissoon S, et al. Atypical pituitary adenomas: incidence, clinical characteristics, and implications. J Neurosurg 2011;114:336-44.

17. Chiloiro S, Doglietto F, Trapasso B, et al. Typical and atypical pituitary adenomas: a single-center analysis of outcome and prognosis. Neuroendocrinology 2015;101:143-50.

18. Lizzul L, Lombardi G, Barbot M, et al. Long-course temozolomide in aggressive pituitary adenoma: real-life experience in two tertiary care centers and review of the literature. Pituitary 2020;23:359-66.

19. Rindi G, Klimstra DS, Abedi-Ardekani B, et al. A common classification framework for neuroendocrine neoplasms: an International Agency for Research on Cancer (IARC) and World Health Organization (WHO) expert consensus proposal. Mod Pathol 2018;31:1770-86.

20. Asa SL, Ezzat S. Aggressive pituitary tumors or localized

Cite this article as: Liu X, Wang R, Li M, Chen G. Pituitary adenoma or pituitary neuroendocrine tumor: a narrative review of controversy and perspective. Transl Cancer Res 2021;10(4): 1916-1920. doi: 10.21037/tcr-20-3446 pituitary carcinomas: defining pituitary tumors. Expert Rev Endocrinol Metab 2016;11:149-62.

21. Ezzat S, Asa SL, Couldwell WT, et al. The prevalence of pituitary adenomas: a systematic review. Cancer 2004;101:613-9.

22. Fernandez A, Karavitaki N, Wass JA. Prevalence of pituitary adenomas: a community-based, cross-sectional study in Banbury (Oxfordshire, UK). Clin Endocrinol (Oxf) 2010;72:377-82.

23. Daly AF, Rixhon M, Adam C, et al. High prevalence of pituitary adenomas: a cross-sectional study in the province of Liege, Belgium. J Clin Endocrinol Metab 2006;91:4769-75.

24. Agustsson TT, Baldvinsdottir T, Jonasson JG, et al. The epidemiology of pituitary adenomas in Iceland, 1955-2012: a nationwide population-based study. Eur J Endocrinol 2015;173:655-64. 